\title{
IMMUNOGENICITY OF SARS-CoV: THE RECEPTOR-BINDING DOMAIN OF S PROTEIN IS A MAJOR TARGET OF NEUTRALIZING ANTIBODIES
}

\section{Yuxian $\mathrm{He}^{*}$}

\section{INTRODUCTION}

The global emergency of severe acute respiratory syndrome (SARS) was caused by a new coronavirus (SARS-CoV) within the family Coronaviridae. ${ }^{1}$ Similar to other CoVs, SARS-CoV is an enveloped virus containing a large, positive-stranded RNA genome that encodes viral replicase proteins and four major structural proteins including spike (S), membrane $(\mathrm{M})$, envelope $(\mathrm{E})$, and nucleocapsid $(\mathrm{N})$. All these proteins and other uncharacterized components can induce immune responses during viral infection. Therefore, post-genomic characterization of the SARS-CoV is highly important for understanding its pathogenesis and for developing diagnostics, therapeutics, and vaccines.

The $\mathrm{S}$ protein of SARS-CoV is a type I transmembrane glycoprotein containing putative S1 and S2 subunits. A fragment located in the middle region of the S1 subunit (residues 318-510) has been characterized as a minimal receptor-binding domain (RBD), which is sufficient to associate with angiotensin-converting enzyme 2 (ACE2), a functional receptor on targeted cells. ${ }^{2}$ The $\mathrm{S} 2$ subunit containing a putative fusion peptide and two heptad repeat (HR1 and HR2) regions is responsible for fusion between viral and target cell membranes. ${ }^{3}$ Therefore, SARS-CoV infection is initiated by attachment of the $\mathrm{S}$ protein via its RBD to the specific receptor on target cells, then forming a fusogenic core between the HR1 and HR2 regions in the S2 domain to bring the viral and target cell membrane into close proximity, resulting in virus fusion and entry.

A second major property of SARS-CoV S protein is that it induces neutralizing antibodies and protective immunity, thereby is a candidate for the development of an effective vaccine. ${ }^{4,5}$ A DNA vaccine encoding the $\mathrm{S}$ protein can induce SARS-CoV neutralization and protective immunity in mice. ${ }^{6}$ Vaccination of animals with recombinant viruses, such as attenuated vaccinia virus (MVA) and parainfluenza virus (BHPIV3), that express $\mathrm{S}$ protein can elicit neutralizing antibodies that protect animals against SARS-CoV challenge. ${ }^{7,8}$ Infection by pseudovirus expressing the SARS-CoV S protein can be effectively neutralized by convalescent sera from SARS patients. ${ }^{9}$ These

*New York Blood Center, New York, New York 10021. 
data suggest that the $\mathrm{S}$ protein of SARS-CoV is a protective antigen, although its antigenic properties have not been well defined. We have recently demonstrated that the $\mathrm{RBD}$ of SARS-CoV S protein is a major target of neutralizing antibodies during viral infection and immunization, ${ }^{10-14}$ and suggested its potential application as a major target for SARS vaccines and immunotherapeutics.

\section{IDENTIFICATION OF NON-NEUTRALIZING EPITOPES IN S PROTEIN}

The S protein of SARS-CoV induces antibody responses in infected patients and in mice and rabbits immunized with the inactivated SARS-CoV. ${ }^{11,12}$ To define its immunoepitopes, a set of 168 peptides spanning the entire sequence of the S protein of SARS$\mathrm{CoV}$ strain TOR2 (each peptide contains 17 amino acid residues with 9 residues overlapping with the adjacent peptides) were synthesized and used in the Pepscan analyses against the convalescent sera from SARS patients. It revealed that the S protein contained five linear immunodominant sites corresponding to the sequences of residues 9-71, 171-224, 271-318, 528-635, and 842-913 (designated as sites I to V). The immunodominant site I-III, and V reacted with more than $50 \%$ of the convalescent sera from SARS patients, and the site IV was reactive with more than $80 \%$ of SARS sera. All of 168 overlapping peptides were also used as probes to localize the immunodominant epitopes of S protein in the SARS-CoV-immunized mice and rabbits. Interestingly, only three of them (536-552, 544-560 and 603-619) reacted significantly with the mouse antisera, and all of them reside within the immunodominant site IV. Similarly, the peptide 536-552 corresponding to the N-terminal sequence of the site IV reacted with all the rabbit antisera. Therefore, the major linear immunodominant domain (the site IV) induces antibody response not only in humans, but also in mice and rabbits. To investigate whether these immunodominant epitopes induced neutralizing antibodies, we designed and synthesized 5 longer peptides that overlap the immunodominant sites based on the above finding. Peptide $\mathrm{S}_{19-48}$ and $\mathrm{S}_{278-312}$ were derived from the immunodominant sites I and III, respectively, while the peptides $\mathrm{S}_{511-552}, \mathrm{~S}_{536-566}$ and $\mathrm{S}_{603-634}$ overlapped with sequence of the major immunodominant site IV. All longer peptides (without conjugation to carrier) were able to elicit high titers of antibodies. However, none of rabbit antisera had neutralizing activity against SARS-CoV or SARS pesudovirus, suggesting that these linear immunodominant sites are not neutralizing epitopes.

\section{IDENTIFICATION OF S PROTEIN RBD AS A MAJOR TARGET OF NEUTRALIZING ANTIBODIES}

We found that convalescent sera from SARS patients contained high titers of RBDspecific antibodies. ${ }^{14}$ To identify neutralizing epitopes of SARS-CoV S protein, a fusion protein containing the RBD (residues 318-510) linked to human IgG1 Fe fragment (designated RBD-Fc) was used to isolate RBD-specific antibodies by immunoaffinity chromatography from patient antisera. ${ }^{14}$ The efficiencies of depletion and recovery of the RBD-specific antibodies were monitored by measuring the reactivity of the starting sera, the corresponding flowthroughs and eluted antibody fractions by ELISA against the RBD and S1 subunit. Neutralizing activities of the samples of the starting sera, flowthroughs and eluates were determined using SARS pseudovirus system. Strikingly, the neutralizing 
activity of immune sera was dramatically reduced after depletion of anti-RBD antibodies, while anti-RBD antibodies in the eluates possessed higher potency than the antibodies in the flowthroughs to neutralize SARS pseudovirus, suggesting that more than $50 \%$ neutralizing activity in the antisera was contributed by the RBD-specific antibodies.

We further observed that the RBD of S protein was a major target of neutralizing antibodies in mice and rabbits immunized with an inactivated SARS-CoV vaccine. All mice and rabbits developed high titers of antibodies against the S protein and its RBD after three immunizations. ${ }^{11}$ The mean end-point titers of mouse and rabbit antisera to the RBD were 1:51,200 and 1:25,600, respectively. Both mouse and rabbit antisera could efficiently block binding of RBD-Fc to ACE2. By using pseudovirus bearing the SARS$\mathrm{CoV} S$ protein, we demonstrated that both mouse and rabbit antisera significantly inhibited S protein-mediated virus entry with mean 50\% inhibitory titers of 1:7,393 and $1: 2,060$, respectively. To further elucidate neutralization determinants of the SARS-CoV, the RBD-specific antibodies were isolated by immunoaffinity chromatography from the rabbit antisera. Similarly, the reactivity of anti-RBD antibodies in the rabbit antisera could be efficiently depleted by RBD-Fc affinity column, as the flowthroughs did not bind to RBD-Fc while the eluted anti-RBD antibodies significantly reacted with the RBD-Fc. The neutralizing activity of anti-RBD antibody-depleted rabbit antisera was much lower than the starting sera, while anti-RBD antibodies possessed more potent activity to neutralize SARS pseudovirus. These data suggest that the RBD of S protein is an effective inducer of neutralizing antibodies in immunized animals.

\section{THE RBD OF S PROTEIN CONTAINS MULTIPLE CONFORMATIONAL EPITOPES THAT INDUCE POTENT NEUTRALIZING ANTIBODIES}

To evaluate whether the RBD can serve as an effective vaccine, the RBD-Fc protein was used as an immunogen to immunize mice and rabbits. ${ }^{10,13}$ All animals (mice and rabbits) developed robust antibody responses against RBD-Fc after boosting. The antisera contained high titers of antibodies specific for the RBD as shown by ELISA using S1 subunit as an antigen. The mean endpoint titers of mouse and rabbit antisera to the S1 protein were 1:625,000 and 1:312,500, respectively. The antisera were further tested for their neutralizing activity using two different assay systems, i.e., infection of SARS-CoV in Vero E6 and of SARS pseudovirus in 293T cells expressing ACE2. Strikingly, both mouse and rabbit antisera were able to effectively neutralize live SARS-CoV infection with mean $50 \%$ neutralization titers of 1:15,360. Infection of ACE2-expressing 293T cells by SARS pseudovirus could be potently inhibited.

To characterize the neutralization determinants on the RBD of S protein, we isolated a panel of 27 monoclonal antibodies (mAbs) from the RBD-Fc-immunized mice. Six groups of conformation-dependent epitopes, designated as Conf I-VI, and two adjacent linear epitopes were identified by ELISA and binding competition assays. ${ }^{10}$ The Conf IV and $\mathrm{V}$ mAbs could efficiently block RBD binding to ACE2. Some mAbs reacting with the Conf III and VI partially inhibited interaction between the RBD and ACE2. This suggests that their epitopes may overlap the receptor-binding sites on the RBD or binding of these mAbs to RBD may cause conformational changes of the receptor binding sites, resulting in inhibition of RBD binding to ACE2. These mAbs also had highly potent neutralizing activities against both SARS-CoV and SARS pseudovirus infections. The mAbs that recognize the Conf I, II could not significantly affect the RBD binding with 
ACE2, but also efficiently neutralized SARS-CoV, suggesting they may use a unique mechanism to block infection. These data indicate that the RBD induces neutralizing antibodies specific not only for the receptor-binding sites, but also for other structural conformations. Notably, two mAbs targeting linear sequences had no virus-neutralization ability. Therefore, the RBD of SARS S protein contains multiple conformational epitopes capable of inducing potent neutralizing antibody responses.

\section{CONCLUSIONS}

In summary, we propose to use the RBD of SARS-CoV for developing vaccines and immunotherapeutics, because it is not only a functional domain that mediates virusreceptor binding but also a major target of neutralizing antibodies during virus infection and vaccination. Independent, folded RBD contains multiple neutralizing epitopes and can induce effective protective antibodies.

\section{REFERENCES}

1. P. A. Rota, M. S. Oberste, S. S. Monroe, et al., Characterization of a novel coronavirus associated with severe acute respiratory syndrome, Science 300, 1394 (2003).

2. S. K. Wong, W. Li, M. J. Moore, H. Choe, and M. Farzan, A 193-amino acid fragment of the SARS coronavirus $\mathrm{S}$ protein efficiently binds angiotensin-converting enzyme 2, J. Biol. Chem. 279, 3197 (2004).

3. S. Liu, G. Xiao, Y. Chen, et al., Interaction between heptad repeat 1 and 2 regions in spike protein of SARS-associated coronavirus: implications for virus fusogenic mechanism and identification of fusion inhibitors, Lancet 363, 938 (2004).

4. Y. He and S. Jiang, Vaccine design for severe acute respiratory syndrome coronavirus, Viral Immunol. 18, 327 (2005).

5. S. Jiang, Y. He, and S. Liu, SARS vaccine development, Emerg. Infect. Dis. 11, 1016 (2005).

6. Z. Y. Yang, W. P. Kong, Y. Huang, et al., A DNA vaccine induces SARS coronavirus neutralization and protective immunity in mice, Nature 428, 561 (2004).

7. H. Bisht, A. Roberts, L. Vogel, et al., Severe acute respiratory syndrome coronavirus spike protein expressed by attenuated vaccinia virus protectively immunizes mice, Proc. Natl. Acad. Sci, USA 101, 6641 (2004).

8. A. Bukreyev, E. W. Lamirande, U. J. Buchholz, et al., Mucosal immunisation of African green monkeys (Cercopithecus aethiops) with an attenuated parainfluenza virus expressing the SARS coronavirus spike protein for the prevention of SARS, Lancet 363, 2122 (2004)

9. Y. Nie, G. Wang, X. Shi, et al., Neutralizing antibodies in patients with severe acute respiratory syndromeassociated coronavirus infection, J. Infect. Dis. 190, 1119 (2004).

10. Y. He, H. Lu, P. Siddiqui, Y. Zhou, and S. Jiang, Receptor-binding domain of SARS coronavirus spike protein contains multiple conformational-dependant epitopes that induce highly potent neutralizing antibodies, J. Immunol. 174, 4908 (2004).

11. Y. He, Y. Zhou, P. Siddiqui, and S. Jiang, Inactivated SARS-CoV vaccine elicits high titers of spike protein-specific antibodies that block receptor binding and virus entry, Biochem. Biophys. Res. Commun. 325, 445 (2004).

12. Y. He, Y. Zhou, H. Wu, B. Luo, J. Chen, W. Li, and S. Jiang, Identification of immunodominant sites on the spike protein of severe acute respiratory syndrome (SARS) coronavirus: implication for developing SARS diagnostics and vaccines, J. Immunol. 173, 4050 (2004).

13. Y. He, Y. Zhou, S. Liu, Z. Kou, W. Li, M. Farzan, and S. Jiang, Receptor-binding domain of SARS-CoV spike protein induces highly potent neutralizing antibodies: implication for developing subunit vaccine, Biochem. Biophys. Res. Commun. 324, 773 (2004)

14. Y. He, Q. Zhu, S. Liu, Y. Zhou, B. Yang, J. Li, and S. Jiang, Identification of a critical neutralization determinant of severe acute respiratory syndrome (SARS)-associated coronavirus: importance for designing SARS vaccines, Virology 334, 74 (2005). 